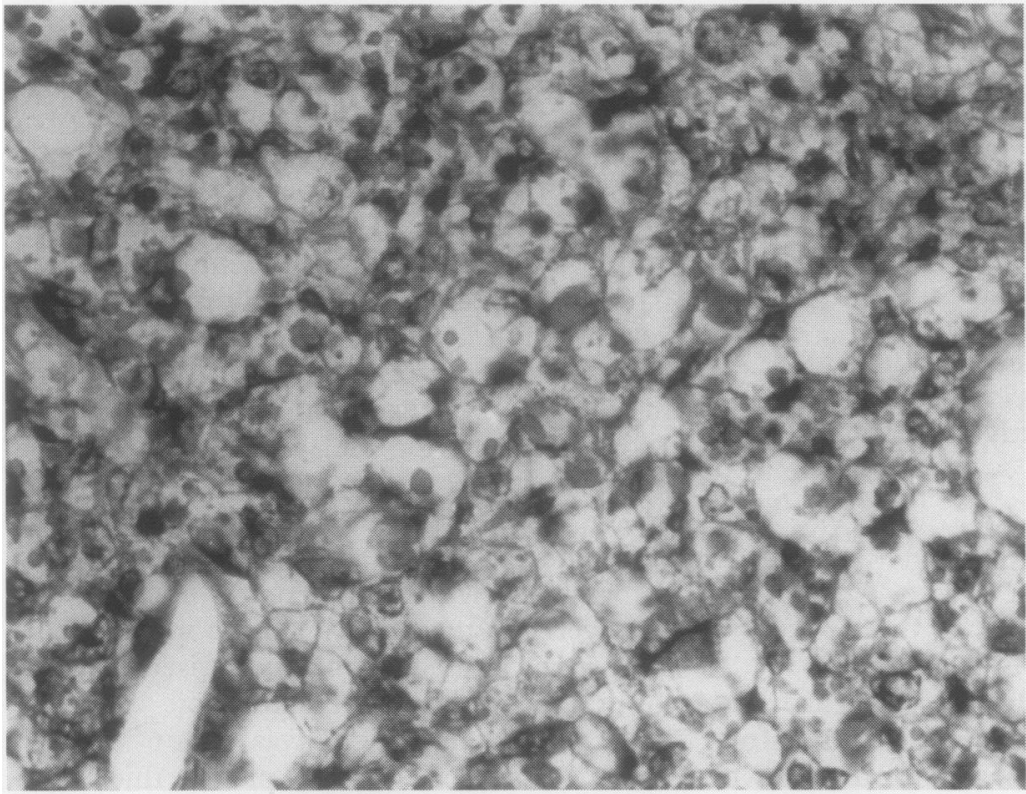

Figure 2 A loose vacuolated network of tumour tissue with hyaline globules both inside and outside the cells. Stain: haematoxylin and eosin. Original magnification $\times 500$.

Endodermal sinus tumours are the most common malignant germ cell neoplasm seen in childhood. They usually originate from the gonads, ${ }^{3}$ but on rare occasions they may be seen in the retroperitoneum, anterior mediastinum, pleura, pericardium, sacrococcyx, central nervous system, liver, and vagina. ${ }^{34}$ The discovery of the metastasis to the peritoneum, pericardium, lymph nodes, and the lungs may be the first manifestation of the tumour. ${ }^{4}$ In our patient there was no evidence of any other lesion.

Despite the fact that malignant tumours are rare causes of spontaneous haemothorax they must not be overlooked in the differential diagnosis. In cases of haemothorax of unknown aetiology thoracotomy may be indicated.

1 Templeton PA, Vanright JR, Rodriquez A, Diaconis JN. Mediastinal tumours presenting as spontaneous haemathorax, simulating aortic dissection. Chest 1988;93:828-30.

2 Klotti J, Walliser G, Sacher P. Nontrauma-induced haemothorax a certain leading symptom of a malignan tumour. $Z$ Kinderchir 1989;44:115-8.

3 Kuzur ME, Cobleigh MA, Greco FA, Einhorn LH, Oldham RK. Endodermal sinus tumour of the mediastinum. Cancer 1982;50:766-74.

4 Truong LD, Harris L, Mattioli C, Hawkins E, Lee A. Endodermal sinus tumour of the mediastinum. Cancer 1986;58:730-9.

\section{Catamenial haemoptysis: a rare} cause

D J Wood, K Krishnan, P Stocks, E Morgan, M J Ward

\footnotetext{
Department of

Medicine

D J Wood

K Krishnan

M J Ward

Department of

Histopathology

P Stocks

Kings Mill Hospital

Mansfield Road,

Sutton in Ashfield

NG17 4JL

Department of

Thoracic Surgery,

City Hospital,

Nottingham NG5 1PB

E Morgan

Reprint requests to:

Dr M J Ward

Received 30 April 1992

Returned to authors

8 June 1992

Revised version received

18 November 1992

Accepted 8 December 1992
}

\begin{abstract}
Since the first report of pulmonary endometriosis as a cause of catamenial haemoptysis all cases have been assumed to be due to pulmonary endometriosis, even in the absence of histopathological proof. A case is presented where the histological findings were of a pulmonary arteriovenous malformation.
\end{abstract}

(Thorax 1993;48:1048-1049)

Periodical haemoptysis occurring in association with the menses (catamenial haemoptysis) is a rare entity. Since the first published case, which was due to endometriosis of the lung, ${ }^{1}$ there have been fewer than 20 reported cases. All the cases described have been attributed to pulmonary endometriosis although less than one third have had histological evidence.

\section{Case report}

A 32 year old woman presented with a 10 month history of recurrent haemoptysis occurring during every menstrual cycle. Symptoms started on the second day of her menses and lasted for three days. The haemoptysis was associated with mild right sided pleuritic type chest pain and never occurred without a period. No other symptoms were noted.

Two years previously she had been treated with methotrexate for nine months for an invasive hydatidiform mole. She had had normal menstruation and serum gonadotrophin levels ever since chemotherapy.

Clinical examination was normal Laboratory investigations revealed normal full blood count, erythrocyte sedimentation rate, urea and electrolyte levels, liver function tests, bone profiles, coagulation studies, and serum gonadotrophin levels. Chest radiography during haemoptysis was normal but fibreoptic bronchoscopy revealed blood originating from the posterior segment of the right upper lobe but no endobronchial lesion was seen. Computed tomography during haemoptysis revealed a fairly well demarcated area of patchy consolidation posteriorly in the right upper lobe. Computed tomography was repeated in the middle of the cycle and revealed a small subpleural nodule at the same site with no evidence of surrounding consolidation or haemorrhage.

The patient was treated by posterior seg- 


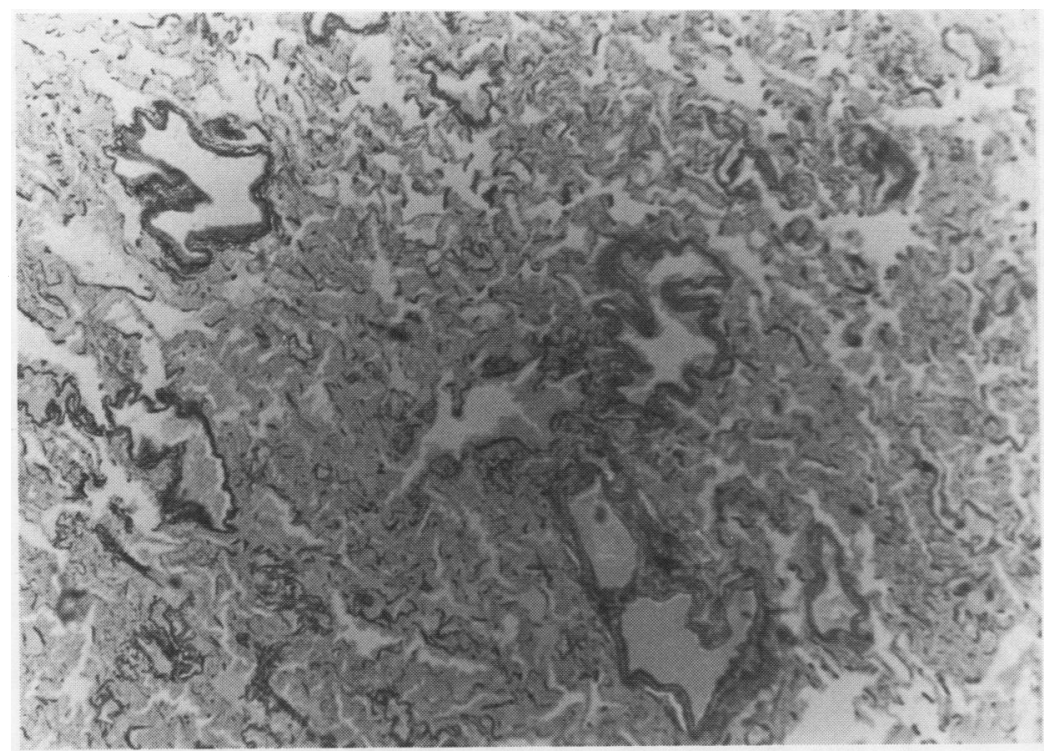

Microscopic section of a lesion removed by segmental resection of the right upper lobe. Van Gieson staining showing numerous blood vessels.

mental resection of the right upper lobe. Histopathological examination of the specimen revealed blood filled alveoli from a presumed intrapulmonary haemorrhage. Numerous vessels were seen in the haemorrhagic area on elastic Van Gieson staining suggesting that this haemorrhage was due to a localised arteriovenous malformation. No evidence of endometriosis, pneumonia or tumour was detected (fig).

The patient made an uneventful recovery with no recurrence of haemoptysis or chest pain.

\section{Discussion}

Catamenial haemoptysis is rare with about 20 cases having been reported since Lattes et al first described cyclical haemoptysis associated with the menses due to pulmonary endometriosis. ${ }^{1}$ All of the cases reported have been attributed to pulmonary endometriosis although histological confirmation has been obtained in less than one third. Previously published guidelines have suggested that the diagnosis of catamenial haemoptysis due to pulmonary endometriosis should be made on the basis of the clinical picture of cyclical haemoptysis associated with the menses after exclusion of other causes of recurrent haemoptysis. ${ }^{2}$

Several techniques have been used in the investigation of catamenial haemoptysis. All of the reported cases had chest radiographs taken during haemoptysis but only a few had a discernible lesion and, when an intermenstrual chest radiograph was taken, the lesion was noted to be smaller or not visible. All but one patient underwent bronchoscopic exami- nation but an endobronchial lesion was identified in only one case. A tissue diagnosis was obtained in two cases, one following transbronchial biopsy ${ }^{3}$ and the other a cytological diagnosis after bronchial lavage. ${ }^{4}$ Computed tomography was performed in six patients; in all cases scans performed during haemoptysis revealed a lesion but it was unusual for intravenous injection of contrast medium to produce enhancement. ${ }^{5}$ Angiography has been previously reported as being of little value in the investigation of patients with catamenial haemoptysis. ${ }^{6}$

Possible theories of the mechanisms by which arteriovenous malformations cause catamenial haemorrhage may be based on the cyclical changes of oestrogens. It has been shown that high doses of oestrogens reduce bleeding from the vascular malformations in hereditary haemorrhagic telangiectasia and that this effect may be due to oestrogens maintaining the continuity of vascular endothelium or by an effect on blood coagulation. ${ }^{7-9}$ Fluid shifts associated with oestrogen induced fluid retention and the natriuretic effects of progesterone may cause changes in pulmonary artery pressure and thus provoke haemorrhage from an arteriovenous malformation.

It has been stated that, with a good history of cyclical haemoptysis occurring in association with the menses and radiographic evidence of a lesion whose character and size changes during and between menses, the diagnosis of pulmonary endometriosis can be made without further investigation. ${ }^{210} \mathrm{We}$ believe our case suggests that arteriovenous malformations should be considered in catamenial haemoptysis.

1 Lattes R, Shepard F, Tovell H, Wylie R. A clinical and pathologic study of endometriosis of the lung. Surg Gynecol Obstet 1956;103:552-8.

2 Guidry GG, George RB. Diagnostic studies in catamenial haemoptysis. Chest 1990;98:260-1.

3 Suginami H, Hamada K, Yano K. A case of endometriosis of the lung treated with danazol. Obstet Gynecol 1985;66(Suppl):685-715

4 Johnson III WM, Tyndal CM. Pulmonary endometriosis: treatment with danazol. Obstet Gynecol 1987;69:506.

5 Hertzanu Y, Hernier D, Hirsch M. Computed tomography of pulmonary endometriosis. Comput Radiol 1987; 11:81-4.

6 Katoh O, Yamada H, Aoki Y, Matsumoto S, Kudo S Utility of angiograms in patients with catamenia haemoptysis. Chest 1990;98:1296-7.

7 Menefee M, Flessa H, Glueck H, Hogg S. Hereditary haemorrhagic telangiectasia. Arch Otolaryngol 1975;101: 246-51.

8 van Cutsen E, Rutgeerts P, Vantrappen G. Treatment of bleeding gastrointestinal vascular malformations with oestrogen-progesterone. Lancet 1990;335:953-5.

9 Livio M, Manucci PM, Vigano G, Mingardi G, Lombardi $R$, Mecca $G$, et al. Conjugated estrogens for the management of bleeding associated with renal failure. $N$ Engl ₹ Med 1986;315:731-5.

10 Elliot DL, Barker AF, Dixon LM. Catamenial haemoptysis: new methods of diagnosis and therapy. Chest 1985;87:687-8. 